\title{
Pratiques
}

Linguistique, littérature, didactique

$159-160 \mid 2013$

Le figement en débat

\section{Le défigement de phrasèmes pragmatiques et sa traduction}

\section{Caroline Pernot}

\section{(2) OpenEdition}

\section{Journals}

Édition électronique

URL : http://journals.openedition.org/pratiques/2900

DOI : $10.4000 /$ pratiques. 2900

ISSN : 2425-2042

Éditeur

Centre de recherche sur les médiations (CREM)

Édition imprimée

Date de publication : 15 décembre 2013

Pagination : 179-188

\section{Référence électronique}

Caroline Pernot, « Le défigement de phrasèmes pragmatiques et sa traduction », Pratiques [En ligne], 159-160 | 2013, mis en ligne le 30 juin 2016, consulté le 19 avril 2019. URL : http:// journals.openedition.org/pratiques/2900 ; DOI : 10.4000/pratiques.2900

(c) Tous droits réservés 


\section{Le défigement de phrasèmes pragmatiques et sa traduction}

\section{Caroline Pernot}

Université de Lorraine, CREM (EA 3476)

\section{Introduction}

Les phrasèmes pragmatiques sont des expressions figées dont la caractéristique est de présenter un sens solidaire de la situation dans laquelle ils sont énoncés. Sous l'impulsion notamment de la didactique des langues et de la traductologie, il est apparu que les phrasèmes pragmatiques présentaient des particularités distinctives qui, de plus, ont amené à un élargissement du champ de la phraséologie, axée à ses débuts sur les aspects morphosyntaxiques et sémantiques des expressions figées (Legallois et Tutin $2013: 5$ ). Ils représentent toutefois un ensemble dont les contours varient selon les critères retenus, définitoires et périphériques (Hyvärinen 2011), aboutissant à une terminologie variable (kommunikative Phraseologismen (Burger 2007), formules de routine / Routineformeln (Coulmas 1981 ; Klein et Lamiroy 2011) ou routines conversationnelles (Bidaud 2002) ou encore phrasèmes situationnels).

L'objet de notre contribution est le défigement, compris comme phénomène discursif qui peut accompagner l'emploi de phrasèmes, et son examen à la lumière de la traduction. Reposant sur un socle contrastif français-allemand, notre démarche se situe néanmoins dans une perspective traductologique, puisqu'il s'agit de s'interroger sur l'équivalence en discours et non en langue des phrasèmes. La confrontation entre textes français et textes allemands et l'analyse des invariants traductologiques sera un révélateur des procédés et des visées du défigement.

\section{Le défigement de phrasèmes pragmatiques}

\subsection{Définition et délimitation}

1.1.1. Les phrasèmes pragmatiques, à la différence des phrasèmes descriptifs, présentent un sens qui ne peut être décrit qu'à travers l'action qu'ils permettent de réaliser. En guise d'exemple, le sens de « Le monde est petit» (en tant qu'expression figée, sachant qu'une forme homonymique ayant un sens référentiel compositionnel peut bien évidemment être énoncée) inclut la situation dans laquelle il s'emploie et 
l'acte illocutoire réalisé, en l'occurrence la manifestation d'une surprise lors d'une rencontre avec une personne qu'on ne s'attendait pas à retrouver en ces lieux. Cette particularité des phrasèmes pragmatiques est une caractéristique ne se confondant pas avec le fait que tout phrasème permet de réaliser un acte illocutoire (Burger / Buhoger / Sialm 1982 : 105). L'imbrication de la situation d'énonciation dans la valeur sémantique d'un phrasème pragmatique se vérifie par l'impossibilité de reformuler au mode indirect un emploi en discours direct. Dans les énoncés suivants, les phrasèmes sont représentés en a) au discours direct, qui forme un cadre énonciatif distinct et autonome par rapport à celui de l'énonciateur rapporteur. Dans les reformulations en b), ces phrasèmes sont placés dans un autre cadre énonciatif, la représentation de discours au mode indirect supposant la réduction à un seul cadre énonciatif :

a) Il m'a répondu : « Tu m'en diras tant. » b) *Il m'a répondu que je lui en dirais tant.

a) Il m'a répondu : «Tu parles... »

b) * Il m'a répondu que je parlais.

a) Il m'a répondu : «Et comment!»

b) * Il m'a répondu que et comment. ${ }^{(1)}$

Par ailleurs, à la différence des phrasèmes descriptifs dont l'emploi peut être au service de la réalisation de différents actes illocutoires (par ex. " $\mathrm{N}$ voit la vie en rose »), les phrasèmes pragmatiques sont monopragmiques (Martins-Baltar 2006 : 301 ) et présentent une valeur illocutoire figée. Enfin, il faut noter qu'ils présentent un degré de compositionnalité ainsi qu'une opacité sémantique gradués («on n’est pas sorti de l'auberge » $v s$ « parlons peu mais parlons bien », « les carottes sont cuites » $v S$ « quand on parle du loup... »).

1.1.2. Dans le cadre de la présente analyse, nous avons également sélectionné des phrasèmes dont le sens est certes lié à la situation d'énonciation mais dont le sémantisme est de nature descriptive. Nous les retenons au motif que dans leurs emplois, leur valeur pragmatique, certes secondaire, est néanmoins solidaire d'une situation d'énonciation et qu'ils se sont figés comme formules de routine, présentant de ce fait une proximité avec les phrasèmes pragmatiques. Notre démarche consistant à inclure ce que nous nommerons des phrasèmes exclusivement pragmatiques (1.1.1) ainsi que des phrasèmes d'emploi pragmatique (1.1.2) est similaire à celle choisie par Klein et Lamiroy (2011). Toutefois, à la différence de ces auteurs, nous incluons dans la catégorie des phrasèmes d'emploi pragmatiques les phrasèmes dont le sujet peut être un sujet nominal libre (du type « N n'est pas la mer à boire » ou « $\mathrm{N}$ me fait une belle jambe ») et l'objet affecté une tierce personne. Nous n'opérons donc pas de distinction entre les routines conversationnelles propositionnelles et infra-propositionnelles. Le phrasème «N peut toujours courir » dans un emploi à la $3^{\mathrm{e}}$ personne (cf. infra 2.2) fait ainsi partie de notre corpus ; dans cette réalisation morphologique, nous voyons une forme délocutive, en nous référant à la définition d'un syntagme délocutif proposée par Anscombre: « [son] sens désigne des objets, des propriétés, des relations ou des actions par référence à un acte illocutoire que 1 'on peut accomplir en énonçant la formule $X$ ! d'où [le syntagme en question] est formellement dérivé. Selon cette définition, la délocutivité lexicale est la création à partir de la valeur pragmatique d'une énonciation, d'une valeur sémantique descriptive. » (Anscombre $1980: 24$ ).

(1) L'étude approfondie des phrasèmes pragmatiques n'étant pas l'objet du présent article, nous exposons ici les caractéristiques les plus saillantes. Le test du discours indirect n'est toutefois pas systématiquement probant: ? Il m'a répondu que c'était le cas de le dire. Il m'a répondu que les bras lui en étaient tombés. De plus, il conviendrait de dissocier ce qui est lié aux caractéristiques des phrasèmes pragmatiques de ce qui a trait de manière générale au discours indirect (impossibilité d'une représentation d'énoncés elliptiques, transfert de la valeur exclamative vers le discours cadre, ...). 


\subsection{Le défigement et sa traduction}

Les phrasèmes étudiés ici relèvent d'un emploi lié à un défigement « métalinguistique » (Mejri 2011 : 74). Ces occurrences se situent dans une tension discursive générée par l'existence formelle et sémantique d'une préconstruction qui se trouve défigée afin que le phrasème soit réinvesti de son sens compositionnel. La convocation du sens compositionnel fait apparaître une double lecture du phrasème, dont l'une repose sur le sens préconstruit et conventionnel et l'autre se situe au moment de l'énonciation. L'acte individuel de parole extrait le phrasème de la sphère collective pour le placer dans un contexte qui le charge d'un sens accessible par les règles interprétatives des compositions libres.

Dans la perspective traductologique, le critère principal de l'équivalence sera celui de l'invariance de la double lecture. Loin de toute recherche d'exhaustivité, nous cherchons à illustrer différents schémas d'équivalence entre les textes français sources et les textes allemands cibles. En outre, un paramètre important conditionnant l'équivalence sera celui de l'inscription dans le texte : nous verrons en effet que plusieurs types d'éléments contextuels peuvent générer la double lecture et nous serons attentifs à leur maintien dans le texte cible. Le défigement et la double lecture peuvent soit être circonscrits à l'emploi du phrasème, soit être prolongé dans le contexte immédiat. Enfin, la caractéristique des phrasèmes pragmatiques d'être souvent employés comme des chevilles de l'interaction, marquant à eux seuls une réaction à un propos ou à un acte, nous amène à tenir compte du paramètre de la cohérence de l'interaction dans l'équation traductologique.

\section{Analyses de traductions}

\section{1. Ça me fait une belle jambe}

Le phrasème « Ça me fait une belle jambe » est employé pour exprimer de l'indifférence vis-à-vis d'un propos ou d'un acte dont on dénie l'utilité ou l'intérêt. Avant que le phrasème ne se lexicalise, l'expression désignait les habits dont les hommes - exclusivement - pouvaient se parer. Elle référait par synecdoque à un homme de bonne condition, comme en témoigne l'occurrence suivante de 1794 :

(1) Madame Desparbès, couchant avec Louis XV, le roi lui dit: « tu as couché avec tous mes sujets. — ah ! Sire. — tu as eu le duc de Choiseul. — il est si puissant ! — le maréchal de Richelieu. — il a tant d'esprit ! — Monville. — il a une si belle jambe ! —à la bonne heure ; mais le duc d'Aumont, qui n'a rien de tout cela. — ah ! Sire, il est si attaché à votre majesté ! » (Chamfort, Caractères et anecdotes, 129)

L'expression évolua ensuite vers le syntagme « faire la belle jambe » pour désigner un comportement de coquetterie galante, toujours appliquée exclusivement à des hommes :

(2) Je venais de faire quelques pas à peine sur le pavé délabré, quand tout à coup, du calle Scuola Dei Fabbri, je vis déboucher la grotesque figure du prince Serge Labounoff, vieux compagnon de débauche du temps que je faisais la belle jambe à la cour de la Sémiramis du nord. (Milosz, L'Amoureuse initiation, 43)

Le phrasème actuel, lexicalisé au XIX ${ }^{\mathrm{e}}$ siècle, procède d'un transfert métaphorique au profit d'une expression désignant par ironie l'indifférence vis-à-vis d'un élément dont l'intérêt n'est pas réel. Actuellement, le lien entre l'image source (« la belle 
jambe ») et le sens phraséologique n'est pas transparent et le phrasème est uniquement perçu comme imagé(2).

Dans les usages défigés que nous avons relevés, nous observons que la remotivation sémantique peut déplacer le sens compositionnel vers un objet féminin, comme l'atteste cet échange consigné dans le Journal de Jules Renard :

(3) 10 avril.

—Ça me fait une belle jambe !

— Madame, vous n'aviez pas besoin de ça. (Renard, Journal : 1887-1910,655)

Dans l'occurrence suivante, le phrasème est employé dans un contexte de discours rapporté, dans lequel le narrateur rapporte le discours non proféré du personnage. Un mouvement du corps trahit le défigement et l'interprétation compositionnelle que le locuteur cité fait du phrasème qu'il emploie :

(4) (une mère reproche à son fils de médire d'elle dans la famille)

- C'est inexact, ma mère. Grand'mère ne parlait jamais de vous et mademoiselle nous a toujours ordonné de prier, pour vous, le matin et le soir.

Mme Rezeau n'osa pas dire : «ça me fait une belle jambe ! ", mais, par suite d'une silencieuse association d'idées, elle se caressa longuement le tibia.

(Bazin, Vipère au poing, 94)

Le traducteur ne dispose pas en allemand de phrasèmes manifestant l'indifférence qui soit construit sur le fonds imagé de la jambe. Afin de conserver la cohérence de la scène représentée, le traducteur s'est orienté vers un phrasème équivalent sur le plan de l'illocution, « das war bestimmt für den Arsch » (littéralement : «c'était sûrement pour le cul »), puisqu'il témoigne de l'indifférence en réaction à un propos ou une action jugée non utile, ce qui nécessite toutefois un changement de registre de langue. Ce fonds imagé permet de faire réaliser au locuteur un geste en concordance avec ce phrasème : « sie strich sich lange über die Seite ihres Gesäßes » (littéralement : « elle se frotta longuement la fesse »).

(5) „Das stimmt nicht, Mutter. Großmutter sprach nie von Ihnen, und Mademoiselle hat uns immer angehalten, für Sie zu beten, morgens und abends.“

Madame Rezeau wagte nicht zu sagen : „Das war bestimmt für den Arsch !“, aber infolge einer stillschweigenden Gedankenverbindung strich sie sich lange über die Seite ihres Gesäßes. (Bazin, Viper im Würgegriff, 65-66)

Toute traduction étant un choix et une hiérarchisation de traits équivalents, il apparaît ici que le traducteur a trouvé une traduction tout à fait adéquate, en choisissant un phrasème certes non équivalent sur le plan du registre de langue, mais équivalent dans sa fonction pragmatique et dans les possibilités de représentation scénique qu'il offre.

\subsection{Tu peux toujours courir}

Le phrasème «N peut (toujours) courir » signale l'inutilité d'une attente ou d'un effort au vu de l'improbabilité de la réalisation d'un objectif. Le fonds métaphorique de la course symbolise de manière assez transparente l'effort et la durée/l'attente, sans que bien sûr, le but poursuivi soit en relation avec un quelconque effort physique :

(2) Dans la catégorie des phrasèmes métaphoriques, le lien métaphorique entre l'image de départ et l'image d'arrivée est soit perçu par les locuteurs comme motivé, auquel cas le phrasème peut être considéré comme figuratif, soit perçu comme arbitraire, auquel cas le phrasème peut être considéré comme simplement imagé. Cette distinction est celle opérée par Burger (2007) entre bildlich (figuratif) vs bildhaft (imagé). 
(6) C'est l'Ivanhoé de l'imparfait du subjonctif. En plus, il le parle couramment, même à la récréation. " Il me serait agréable que tu me rendisses mon goûter. »Il peut toujours courir. (Picouly, Le champ de personne, 143)

L'extrait suivant montre un défigement articulé autour d'une caractéristique évoquée dans la description du personnage concerné :

(7) (des policiers enquêtent sur la mort d'un marathonien)

Vingt-deux joggers plus loin, ils tombèrent enfin sur le type, un petit maigre qui préparait le marathon de Paris et qui connaissait Élie. [...]

- [...] Il courait vite, Élie. Un vrai pro.

- Qu'est-ce que vous savez sur lui ? Il travaillait ? Il était français ?

- Ben oui, pourquoi ? Il vendait des trucs dans la rue, je crois. Des bijoux, dans le Suquet. [...] Son rêve, c'était de faire le marathon de New York. Pauvre gars, ajoutat-il en regardant encore une fois la photo prise à la morgue, y peut toujours courir, maintenant ! (Aubert, Descente d'organes, 42-43)

Appliqué à un marathonien décédé, le phrasème voit son emploi se défiger et le sens compositionnel affleurer. Pour traduire ce commentaire caustique et chargé d'un double sens, le traducteur ne dispose pas d'un phrasème construit sur la même base métaphorique. Néanmoins, il a maintenu une double lecture avec l'expression « Jetzt ist er aus dem Rennen » (littéralement : « Il n'est plus dans la course, maintenant »), qui certes déplace le sens du phrasème (au lieu de l'inutilité de l'effort, c'est l'échec qui est désigné) mais assure une équivalence discursive.

(8) Zweiundzwanzig Jogger später trafen sie schließlich auf den Richtigen, einen kleinen hageren, der für den Marathonlauf von Paris trainierte und der Elie kannte. $[\ldots]$

„[...] War ein guter Läufer, der Elie. Ein echter Profi.“

„Was wissen Sie sonst noch über ihn? Hat er gearbeitet? Ist er Franzose?"

„Schon. Wieso? Er hat irgendwas auf der Straße verkauft. Schmuck, wenn ich mich nicht irre, im Suquet-Viertel. [...] Sein Traum war es, am Marathon von New York teilzunehmen. Armer Kerl“, fügte er nach einem letzten Blick auf das Foto aus dem Leichenschauhaus hinzu. „Jetzt ist er aus dem Rennen.“(Aubert, Nachtlokal, 42-43)

Dans le texte source suivant, la lecture n'est pas nécessairement double :

(9) (le locuteur quitte son ancien appartement)

Adieu studio, $[\ldots]$ adieu vieille andouille d'évier constamment bouché à l'émeri et constamment débouché à l'aiguille à tricoter, adieu jours avortés, heures enfuies, minutes papillons de nuit, adieu feux de bois que je n'ai jamais faits parce que trouver du bois en ville tu peux toujours courir et que l'arbre de Noël était en plastique, adieu, bon Dieu que le temps passe [...] (Benoziglio, Cabinet portrait, 52)

On peut lire l'énoncé de manière exclusivement phraséologique et le comprendre comme signifiant : « espérer trouver du bois en ville est peine perdue ». Le traducteur a toutefois fait le choix de privilégier le sens compositionnel. Il emploie une locution non phraséologique, « du kannst lange nach N rumlaufen » (littéralement : « tu peux courir longtemps dans tous les sens après $\mathrm{N} »)$, dont le verbe support indique un effort concret ${ }^{(3)}$. En somme, le défigement se situe dans l'entre-deux de la traduction : dans le texte source, la lecture compositionnelle n'est que potentielle, tandis que dans le texte cible, celle-ci a gagné en visibilité.

(3) Il en va de même des verbes « rumtelefonieren » (téléphoner à droite et à gauche) ou « rumschnüffeln» (fouiner dans tous les coins). 
(10) Adieu, Appartement, [...] adieu alter Weichselzopf im ständig verstopften und mit der Stricknadel wieder freigeprokelten Ausguß, adieu verkorkste Tage, vertane Stunden, entflatterte Nachtfalterminuten, adieu Kaminfeuer, die ich nie angezündet habe, denn in der Stadt kannst du lange nach Holz rumlaufen und der Weihnachtsbaum ist eh aus Plastik, adieu, mein Gott wie die Zeit vergeht [...]. (Benoziglio, Porträt-Sitzung, 50)

\subsection{Mon oeil}

Le texte suivant et sa traduction illustrent le cas où le traducteur est confronté à l'absence dans la langue cible d'un phrasème pragmatique construit sur le même fonds imagé. Dans le texte français, le phrasème « mon œil » est employé pour désavouer les propos précédents. Une chaîne de défigement est présente dans le texte, en amont avec « vous voyez ? Je voyais » et en aval avec « et... et votre œil ». S'il existe bien l'expression «sehen Sie ? (littéralement : "vous voyez ?») en allemand, il n'existe pas de phrasème construit sur la métaphore oculaire ayant une fonction illocutoire similaire à « mon œil ». La traduction a procédé à un nivellement de toute la séquence, effaçant l'effet humoristique du défigement, et ne conserve que l'équivalent fonctionnel afin d'assurer la cohérence de l'interaction. Le texte allemand correspond à : «Vous comprenez? Et comment que je comprenais. J'ai fermé définitivement ma gueule. Et... et votre œil ». " Mon œil » trouve une correspondance partielle dans l'énoncé « Bei mir ging endgültig die Klappe runter » (littéralement : «j'ai définitivement fermé ma gueule »), équivalent perlocutoire du phrasème pragmatique du texte source.

(11) (le narrateur, un homme borgne, visite un appartement)

- A propos, poursuit-il, la concierge m'a dit votre nom l'autre jour. C'est d'où, un nom pareil ?

Je bredouille ma petite histoire.

— Tiens ? dit-il. Suisse ? J'aurais cru que... Comprenez, j'ai rien contre les youp... juifs, mais ça la fout mal pour l'immeuble, voyez?

Je voyais, mon œil.

- Et... et votre œil, là, c'est de naissance ou un accident ?

Heureusement, de violents coups frappés contre le mur me dispensent de répondre. (Benoziglio, Cabinet portrait, 73)

(12) - Apropos, fährt er fort, die Concierge hat mir neulich Ihren Namen genannt. Wo kommen Sie her, mit so einem Namen?

Ich stammle meine kleine Geschichte.

— Ach? sagt er. Schweizer? Ich dachte schon, Sie ... Verstehen Sie mich recht, ich habe nichts gegen die Jordanplansch... gegen die Juden, aber die passen hier nicht ins Haus, verstehen Sie?

Und ob ich verstand. Bei mir ging endgültig die Klappe runter.

- Und ... und Ihr Auge da, haben Sie das von Geburt oder war das ein Unfall?

Glücklicherweise erspart mir heftiges Gehämmer gegen die Wand eine Antwort. (Benoziglio, Porträt-Sitzung, 72)

\subsection{Ce n'est pas la mer à boire}

Si l'exemple 7 a montré que le défigement peut reposer sur la présence d'un sème commun entre le contexte et le sens compositionnel résiduel du phrasème, les exemples suivants rappellent que cette condition n'est pas suffisante. Le phrasème « Ce n'est pas la mer à boire » est employé pour marquer une volonté de relativiser une difficulté. Dans l'exemple 13, le discours indirect libre « Il ne lui restait que « trois ou 
quatre ans à tirer », ce ne serait pas la mer à boire ! » est la représentation d'un discours que tient un marin, sans que pour autant le référent de la mer soit convoqué dans son discours ; la traduction est un équivalent fonctionnel qui privilégie l'invariance de l'acte illocutoire réalisé et passe par une expression non phraséologique ( « das sei ja keine Ewigkeit », littéralement : « ce n'est pas une éternité »). L'exemple 16 contient également une occurrence de discours indirect libre dans laquelle le personnage se représente sa vie de futur marchand dans une zone portuaire, comparant son activité à celle des pêcheurs bibliques : dans le texte cible français, la présence de l'image de la mer n'est pas corrélée au défigement et le traducteur l'emploie comme équivalent de la séquence " das wird doch hinzukriegen sein ? », littéralement : " c'est quand même pas un truc impossible ».

(13) Dès sa première lettre, il m'avoua ce qu'il avait refusé de me dire à Mahé : il ne continuait pas le thon aux Seychelles. Son fameux et mystérieux projet d'Afrique du Sud, il allait le réaliser. Il ne lui restait que « trois ou quatre ans à tirer», ce ne serait pas la mer à boire ! Ces gens-là décidément, qui ne connaissent ni les quarante heures, ni les jours fériés, ni le repos hebdomadaire, n'ont pas le même sens de la durée que nous. Trois ans à tirer me paraissaient à moi le bout du monde [...]. (Groult, Les vaisseaux du cæur, 268)

(14) Schon in seinem ersten Brief gestand er mir, was er mir in Mahé nicht gesagt hatte: Er würde mit dem Thunfisch auf den Seychellen nicht weitermachen. Sein berühmtberüchtigtes, mysteriöses Projekt in Südafrika würde er nun doch verwirklichen. Es blieben ihm nur noch drei oder vier Jahre durchzuhalten — das sei ja keine Ewigkeit. Leute, die weder die Vierzig-Stunden-Woche noch Feiertage noch Wochenendruhe kennen, haben ganz ersichtlich nicht die gleiche Auffassung von Zeit und Dauer wie wir. Drei Jahre durchhalten, das war für mich eine endlose Zeit [...]. (Groult, Salz auf unserer Haut, 257)

(15) Jetzt kommt die Hafenbahn herangeschnauft mit einem Klingelmann vorweg, zu Fuß, und der Lokomotivführer guckt aus dem Fenster, ob er dem Klingelmann nicht auf die Hacken fährt. Kling-bingl Kling-bingl so macht das, und Karl kann sich erinnern : Ja, das hat er als Kind auch gern gehört. Das war als Kind immer schon so schön. Aber : so eine große Maschine und nur ein einziger Waggon?

Kaufmann zu sein, das denkt er sich leicht. Wie die Fischer in Graal : erst ist das Netz leer, dann voll. Man sitzt am Schreibtisch, diktiert Briefe und telephoniert. Geschäfte machen, das wird doch hinzukriegen sein? (Kempowski, Aus großer Zeit, 429-430)

(16) Le train portuaire arrive à ce moment en haletant, précédé des coups de sonnette d'un homme dont le conducteur de la locomotive, depuis sa fenêtre prend bien soin de ne pas écraser les talons. Cela fait Kling-bing ! Kling-bing !, et Karl peut se souvenir : oui, tout enfant déjà, il aimait à entendre ça. C'était déjà si beau pour l'enfant qu'il était. Mais : une si grosse machine pour un seul wagon?

Il s'imagine qu'être marchand, c'est facile. Comme les pêcheurs à Graal : les filets sont d'abord vides, et ensuite pleins. On est à son bureau, on dicte des lettres, on téléphone. Faire des affaires, ce n'est pas la mer à boire? (Kempowski, Les temps hérö̈ques, 267-268)

Dans le texte suivant, le phrasème apparaît sous une forme tronquée, tant sur le plan formel qu'illocutoire : employé à l'affirmatif et réduit à un syntagme nominal, le phrasème est détourné vers un emploi purement descriptif. Sa remotivation sémantique, associant « la mer à boire » et la comparaison des couleurs de la mer à celles du vin, se fait de plus dans un mouvement de dédoublement énonciatif : le narra- 
teur-locuteur emploie le phrasème défigé, tandis que l'énonciateur, la sphère collective, convoqué par la question rhétorique «ne dit-on pas?», emploie virtuellement le phrasème «ce n'est pas la mer à boire ».

(17) Nous revenons sur le pont. Main en visière, Abel scrute l'horizon. Dans la lumière fatiguée du jour, la mer a les couleurs fortes d'un bon vin. Ne dit-on pas «la mer à boire »?

«Ils ne devraient plus tarder maintenant ! » remarque le bosco.

D'ailleurs, le quai s'anime, les chariots ont été approchés pour recevoir les caisses de poisson. (Boissard, Marie-Tempête, 27)

En allemand, il ne s'est pas forgé d'expression reposant sur un fonds imagé similaire, de sorte que le texte cible, dans lequel a été maintenue la métaphore ainsi que le renvoi à une supposée préconstruction du discours, produit un effet d'étrangeté.

(18) Wir gehen wieder auf Deck. Mit der Hand über den Augen sucht Abel den Horizont ab. In dem müden Tageslicht hat das Meer die kräftige Farbe eines guten Weines. Sagt man nicht ,ein Meer zum Trinken“?

„Sie werden bald zurück sein !“, bemerkte der Bootsmann.

Auf dem Kai wird es lebendig, die Karren sind näher gebracht worden, um die Kisten mit Fisch aufzunehmen. (Boissard, Der Ruf des Meeres, 27)

\subsection{Un ange passe}

Ce phrasème est employé pour relever le fait qu'un silence s'est installé et souligner l'embarras qui est à son origine. Or, si cette situation sociale est conventionnellement associée à un phrasème en français, elle n'appelle pas en allemand de formule conventionnelle. Le défigement du phrasème rend la métaphore de l'ange vivace et la convoque aux fins d'une comparaison avec l'image du Manekenpiss. Si la traduction avait fait le choix d'une équivalence respectant uniquement la cohérence narrative (du type « un silence gêné s'installa»), elle aurait perdu le socle sur lequel repose la référence à l'élément culturel que représente le Manekenpiss. Face à ces difficultés, le traducteur adopte une stratégie se situant aux limites de la traduction : il procède à une transcription littérale accompagnée d'un renvoi à l'expression idiomatique du texte source (littéralement : «Un ange passe. NdT : Expression française lorsqu'un silence soudain apparaît dans une conversation »).

(19) «Tu oublies kekchose », dit maman. Non, papa n'oublie rien mais il préfère passer sous silence l'épisode auquel maman fait allusion. Hélas! Déjà les dames sont intriguées. Papa a beau secouer la tête et lancer des regards implorants, elles insistent : «Allons, monsieur Joseph, ne soyez pas modeste, dites-nous ce qui s'est passé. » Tandis que papa fixe le bout de ses chaussures, maman lève le secret : «II est pas moteste, il est fier. Il veut pas tire qu'il a fait bibi dans sa culotte ! » Un ange passe, qui a des grâces de Manekenpiss. Les dames toussotent pour dissiper la gêne et conviennent qu'à la place de papa, elles en auraient fait tout autant. (Egen, Les tilleuls de Lautenbach, 126)

(20) „Etwas hast du vergessen“, sagt Mama. Wieso denn, Papa vergißt nie etwas, jedoch verschweigt er, was Mama meint. Die Damen geben sich lustern auf das Detail. Papa windet sich, wirft bittende Blicke, sie wollen es wissen : „Na, Monsieur Joseph, sagen Sie uns doch, was dann geschehen ist“. Papa betrachtet höchst verlegen seine Schuhspitzen, und Mama lüftet das Geheimnis : Joseph ist zu stolz, um es zu sagen, nämlich, daß er vor Angst in die Hose gemacht hat! Ein Engel schwebt vorbei ${ }^{1}$ und hat etwas von der Grazie des Manekenpiß. Die Damen hüsteln leicht und geben 
dann zu, an Papas Stelle wäre ihnen wohl das gleiche passiert. (Egen, Die Linden von Lautenbach, 105)

1 Französischer Ausdruck Wenn in einer Gesprächsrunde plötzliches Schweigen eintritt

\section{Conclusion}

Les différents cas illustrés ici montrent des degrés de complexité divers. Plusieurs stratégies de traduction ont été mises en œuvre, tenant compte du maintien de la valeur illocutoire des phrasèmes pragmatiques dans le texte source ou de son effacement complet au profit du seul fonds métaphorique. Si nous retenons comme critère d'équivalence l'emploi pragmatiquement comparable et la double lecture, nous observons que certaines stratégies amènent à une absence de réelle équivalence, lorsque la traduction recourt à un calque - produisant un effet d'étrangeté ou se dévoilant comme telle dans le paratexte traductologique - ou qu'elle se situe uniquement dans une équivalence de la cohérence interactionnelle. Néanmoins, des cas d'équivalence quasi complète sont également attestés dans les traductions de « tu peux toujours courir » et de «ça me fait une belle jambe», qui montrent que des emplois défigés de phrasèmes pragmatiques peuvent passer l'épreuve de la transposition dans une autre langue-culture.

\section{Corpus}

Aubert, B., Descente d'organes, Paris, Seuil, 2001. Traduction allemande par Eliane Hagedorn et Barbara Reitz, Nachtlokal, München, Goldmann, 2002.

BAZIN, H., Vipère au poing. Paris, Grasset, 1948. Traduction allemande par Johannes Hübner, Viper im Würgegriff. Berlin, Verlag der Nation (RDA), 1956.

Benoziglio, J.-L., Cabinet portrait. Paris, Seuil, 1980. Traduction allemande par Claus Sprick, Porträt-Sitzung. Zürich, Benziger / Ex Libris, 1990.

BOISSARD, J., Marie-Tempête. Paris, Laffont, 1998. Traduction allemande par Angelika Weidmann, Der Ruf des Meeres. München, Knaur, 2002.

Chamfort, Sébastien-Roch Nicolas de, Caractères et anecdotes, Paris, Larousse, 1929.

EGEN, J., Les tilleuls de Lautenbach. Mémoires d'Alsace. Paris, Stock 1979. Traduction allemande par Claude-Gérard Benni, Die Linden von Lautenbach. Eine deutschfranzösische Lebensgeschichte. Hamburg, Rowohlt 1986.

Groult, B., Les Vaisseaux du Cour. Paris, Grasset, 1988. Traduction allemande par Irène Kuhn, Salz auf unserer Haut. München, Knaur, 1992.

KeMPOWsKI, W., Aus großer Zeit. Hamburg, Knaur, 1978. Traduction française par JeanPaul Clerc, Les temps hérö̈ques. Lausanne, Éditions l'Âge d'Homme, 1990.

Milosz, O., L'amoureuse initiation, Paris, Silvaire, 1958.

PiCOUly, D., Le Champ de personne, Paris, Flammarion, 1995.

RENARD, J., Journal : 1887-1910, Paris, Gallimard, 1960. 


\section{Bibliographie}

AlBRECHT, J. (2005), « Nochmals zum Begriff der Übersetzungsäquivalenz. Ein Versuch zur Ausräumung von Missverständnissen ». In : Schmitt, C. und Wotjak, B. (Hg.), Beiträge zum romanisch-deutschen und innerromanischen Sprachvergleich. Bonn, Romanistischer Verlag, 1-11.

Anscombre, A. (1985), « De l'énonciation au lexique : mention, citativité, délocutivité ». Langages, 80, 9-34.

BIDAUd, F. (2002), Structures figées de la conversation. Analyse contrastive français italien. Berne, Peter Lang.

Burger, H. (2007), Phraseologie. Eine Einführung am Beispiel des Deutschen, 3., neu bearb. Aufl. Berlin, Schmidt.

Burger, H., Buhofer, A., Sialm, A. (1982), Handbuch der Phraseologie. Berlin, de Gruyter.

Coulmas, F. (1981), Routine im Gespräch. Zur pragmatischen Fundierung der Idiomatik. Wiesbaden, Athenaion.

HYVÄRINEN, I. (2011), «Zur Abgrenzung und Typologie pragmatischer Phraseologismen - Forschungsüberblick und offene Fragen ». In : Hyvärinen, I. und Liimateinen, A. (Hg.), Beiträge zur pragmatischen Phraseologie. Frankfurt am Main, Peter Lang, 9-43.

KLEIN, J.-R. et LAMIROY, B. (2011), « Routines conversationnelles et figement ». In: Anscombre, J.-Cl. et Mejri, S. (éds.), La parole entravée : études sur le figement. Paris, Champion, 195-213.

Legallois, D. et TUtin, A. (2013), «Présentation : Vers une extension du domaine de la phraséologie », Langages, 189, 3-25.

MARTINS-BALTAR, M. (2006), "Comment constituer une nomenclature en pragmatographie ?»In: Szende, T., Le français dans les dictionnaires bilingues, Paris, Champion, 193-203.

MEJRI, S. (2011), «Figement, collocation et combinatoire libre». In: Anscombre, J.-Cl. et Mejri, S. (éds.), La parole entravée : études sur le figement. Paris, Champion, 6477. 